\title{
Postpartum panhypopituitarism with spontaneous pregnancy
}

\author{
Merces Assumpcao-Morales, Maria Milan, Vinuta Mohan, Tasneem Zahra
}

Department of Endocrinology, Lincoln Medical and Mental Health Center, New York, USA

Email: Meresca@hotmail.com, mariteremilan@gmail.com, Vinuta.Mohan@nychhc.org, Tasneem.zahra@nychhc.org

Received 6 April 2013; revised 6 May 2013; accepted 14 May 2013

Copyright (C) 2013 Merces Assumpcao-Morales et al. This is an open access article distributed under the Creative Commons Attribution License, which permits unrestricted use, distribution, and reproduction in any medium, provided the original work is properly cited.

\section{ABSTRACT}

We are reporting a case of a patient found to be pregnant in the setting of panhypopituitarism. There are an only few reports of spontaneous pregnancy in patients with panhypopituitarism. This is a 26-year-old female who was admitted due to symptomatic hypoglycemia. Past medical history is significant for uncomplicated pregnancy with Csection delivery 1 year before admission. She was not able to breastfeed the baby in postpartum period. Her menses have been irregular since then. She also noticed increasing fatigue, muscle weakness, episodic diarrhea and 70 pounds weight loss. Physical exam was remarkable for hypotension. Work up showed elevated levels of beta-HCG, hyponatremia, normal anion gap metabolic acidosis, normochromic anemia, and low cortisol levels, appropriate low levels of insulin and C-peptide. Hypoglycemia panel revealed undetectable levels of sulphonylureas. Serum levels for pituitary hormones showed low levels of ACTH, prolactin and IGF-1, normal levels of FSH, and inappropriate normal levels of TSH in the setting of decreased T4. Stress doses of hydrocortisone were initiated along with thyroid hormone replacement. Brain MRI was found to be unremarkable. Sheehan's syndrome is a very rare complication of pregnancy when necrosis of the pituitary gland occurs after severe hemorrhage and hypotension upon delivery. Small sella size, disseminated intravascular coagulopathy, stress of the delivery and autoimmunity are also implicated as possible causes of Sheehan's syndrome. We should be very attentive for symptoms as failure to lactate and failure to resume regular menses after delivery as they can be the first manifestation of the disease. Infertility is common in these patients, but in very few of them gonadotropin response will be present due to residual pituitary function and spontaneous pregnancy can occur.
Proper management of these patients during pregnancy will also be very important since a high risk of miscarriages can be expected.

Keywords: Sheehan Syndrome; Pregnancy; Panhypopanpituitarism; Pituitary

\section{INTRODUCTION}

We are reporting a case of a patient found to be pregnant in the setting of panhypopituitarism. The patient had an uneventful pregnancy and C-section delivery one year prior to presentation. After delivery the patient presented with irregular menses and failure to lactate and later, presenting with extreme fatigue and weight loss. There are only few reports of spontaneous pregnancy in patients with panhypopituitarism. Infertility treatment for ovulation induction with higher doses of gonadotropins and longer duration of therapy will be required for those patients that aim to get pregnant.

\section{CASE}

This is a 26-year-old female with unknown significant past medical history who was admitted due to symptomatic hypoglycemia. This patient reported flu-like symptoms the day prior with associated poor appetite, nausea and vomiting. Past medical history significant for uncomplicated pregnancy with C-section delivery 1 year before admission. She was not able to breastfeed the baby in postpartum period. Her menses have been irregular since then. She also noticed increasing fatigue, muscle weakness, with episodic diarrhea and 70 pounds weight loss. Family history is significant for type II diabetes. Physical exam remarkable for hypotension. During the initial work up, the patient presented with elevated levels of beta-HCG which folded in the next 2 days; hyponatremia, normal anion gap metabolic acidosis, normochromic anemia, and low cortisol levels, appropriate low levels of insulin and C-peptide. Hypoglycemia panel 
revealed undetectable levels of Sulphonylureas. Since cortisol levels were low, work up for adrenal insufficiency and serum levels for all pituitary hormones were sent with the following findings: low levels of ACTH, prolactin and IGF-1, normal levels of FSH, and inappropriate normal levels of TSH in the setting of decreased T4. he patient remained clinically stable while in the hospital. Stress doses of hydrocortisone were initiated along with thyroid hormone replacement. At the time patient refused brain imaging studies and opted to continue follow up with her primary medical doctor as an outpatient. She was discharged with thyroid and hydrocortisone replacement therapy and was oriented to follow up with her PCP, endocrinology and gynecology. PCP was contacted at the time; MRI was requested along with the referrals. Brain MRI was found to be unremarkable. Unfortunately she was lost to follow up along with the outcome of her pregnancy and hormonal deficiencies.

\section{DISCUSSION}

Sheehan's syndrome is a rare condition that can occur after pregnancy secondary to necrosis of pituitary gland usually is associated with excessive blood loss during or just after deliver. It's well known that pituitary gland is enlarged during pregnancy as a result of stimulation of lactotroph cells by estrogens produced by the placenta. This hyperplasia of lactotroph cells in the pituitary may result in compression of the superior hypophyseal artery and may cause mild ischemia in the gland [1]. Any insult that will change the pressure in the arteries like severe hypotension and excessive bleeding can worsen the ischemia and lead to necrosis of the gland. Sheehan's syndrome can rarely occur amongst patient with no reported postpartum bleeding or complications. Small sella size, disseminated intravascular coagulopathy, stress of the delivery and auto-immunity are also implicated as possible causes of Sheehan's syndrome [2,3]. The extension of the pituitary necrosis determines the onset of the manifestations (which can be days to many years) and also the magnitude of pituitary hypofunction (since the severity of the hormonal deficiency and the number of affected pituitary hormones can vary). Significant clinical manifestation occurs when $75 \%$ of the gland is compromised. This is the reason that most of these patients have a mild disease that goes undiagnosed for a long time. Earlier manifestations would be failure to lactate and failure to resume regular menses. Hypothyroidism and adrenal insufficiency occur in a later period in the course of disease. As suspicions of this diagnosis arise we should request pituitary hormones levels. If hormonal levels are decreased imaging studies should be requested in order to rule out any possible surgical condition and clarify the diagnosis. MRI usually shows partial or total empty sella but a normal study cannot rule out the diagnosis. In a study that evaluated 28 patients found per CT and/or MRI that 8 pts (28.5\%) had an empty sella and 20 patients (71.5\%) a normal image study [4]. Pituitary adenoma and lymphocytic hypophysis should be kept in mind as a differential diagnosis since they can also occur in postpartum period. It was ruled out in our patient due to absence of mass effect symptoms like visual field impairment and headaches, that is usually common in those conditions and no pertinent MRI findings .Treatment should be initiated upon diagnosis and steroid replacement should be started as soon as possible followed by thyroxin replacement. If thyroid hormone is replaced before cortisol, it will speed the body metabolism as well as cortisol breakdown with a potential chance to induce hypoadrenal crisis. Estrogens and GH replacement should be evaluated individually. Infertility is a common finding in these patients and if pregnancy is warranted gonadotropin treatment should be started in order to induce ovulation. Higher doses and longer therapy with gonadotropin is required. Higher indices of miscarriages have been reported in those patients as well. These rare patients that present with spontaneous pregnancy are noted to have some functional reserve of gonadotropins from any residual pituitary tissue [5].

\section{CONCLUSION}

Sheehan's syndrome is a very rare complication of pregnancy when necrosis of the pituitary gland occurs after severe hemorrhage and hypotension upon delivery. There are few reports of patients with Sheehan's syndrome and uncomplicated pregnancy course. Ischemia of the gland can also be explained by the stress of the delivery, small sella size, DIC and auto-immunity. We should also be very attentive for symptoms as failure to lactate and failure to resume regular menses after delivery as they can be the first manifestation of the disease. $75 \%$ of the pituitary gland needs to be destroyed before clinical manifestations became evident and this can take several days to years. Hypothyroidism and adrenal insufficiency occur in the later period of the disease. Hormonal panel should be ordered as soon as the diagnosis is suspected with replacement of steroids and thyroid hormones as needed in order to avoid overt panhypopituitarism. Infertility is common in these patients, but in very few of them gonadotropin response will be present due to residual pituitary function and spontaneous pregnancy can occur. Proper management of these patients during pregnancy will also be very important since a high risk of miscarriages can be expected.

\section{REFERENCES}

[1] Kelestimur, F. (2003) Sheehan's syndrome. Pituitary, 6, 
81-88. doi:10.1023/B:PITU.0000023425.20854.8e

[2] Goswami, R., Kochupillai, N., Crock, P.A., Jaleel, A. and Gupta, N. (2002) Pituitary autoimmunity in patients with Sheehan's syndrome. The Journal of Clinical Endocrinology \& Metabolism, 87, 4137-4141. doi:10.1210/jc.2001-020242

[3] Shivaprasad, C. (2011) Sheehan's syndrome: Newer advances. Indian Journal of Endocrinology and Metabolism, 15, 203-207. doi:10.4103/2230-8210.84869

[4] Sert, M., Tetik, T., Kirim, S. and Kocak, M. (2003) Clinical report of 28 Patients with Sheehan's syndrome. Endocrine Journal, 50, 297-301. doi:10.1507/endocri.50.297

[5] Ganie, M.A., Laway, B.A., Qazi, S., Jehangir, M., Butt, T.P. and Koul, S. (2010) Spontaneous successful pregnancy in post-surgical hypopituitarism: A case report. Turkish Journal of Endocrinology \& Metabolism, 14, 23

[6] See, T.T., Lee, S.P. and Chen, H.F. (2005) Spontaneous pregnancy and partial recovery of pituitary function in a patient with Sheehan's syndrome. Journal of the Chinese Medical Association, 68, 187-190.
doi:10.1016/S1726-4901(09)70247-3

[7] Zargar, A.H., Masoodi, S.R., Laway, B.A., Sofi, F.A. and Wani, A.I. (1998) Pregnancy in Sheehan's syndrome: A report of three cases. Journal of the Association of Physicians of India, 46, 476-478.

[8] Bhagat, M., Salhan, S., Sarda, N. and Bajaj, B. (2011) Spontaneous pregnancy in a patient with Sheehan's syndrome. JK Science, 13.

[9] Vieira, H.B., Knoepfelmacher, M., Salgado, L.R., Wajchenberg, B.L. and Liberman, B. (1995) Preservation of gonadotrophic function and pregnancy in Sheehan's syndrome: A case report and review of the literature. Revista da Associação Médica Brasileira, 41, 135-138.

[10] Nadia, M., AliInsaf, H., Karima, K., Youssef, L. and AbdallahNeji, B. (2010) Spontaneous pregnancy in Sheehan syndrome. Endocrine Abstract, 22, 490.

[11] Daniel, A., Ezzat, S. and Greenblatt, E. (2012) Adjuvant growth hormone for ovulation induction with gonadotropins in the treatment of a woman with hypopituitarism. Case Reports in Endocrinology, 2012. doi:10.1155/2012/356429 